\title{
Transoral laser resection of a large schwannoma in the retropharyngeal
}

\section{space}

\section{Payman Dabirmoghaddam ${ }^{1}$, Zahra Danesh ${ }^{2 *}$ and Amirmohammad Danesh ${ }^{3}$}

\author{
${ }^{1}$ Associate Professor of Otolaryngology, Tehran University of Medical Science, Amiralam Hospital, \\ Tehran, Iran \\ 2Otolaryngologist,Shahrekord University of Medical Science, Kashani Hospital, Shahrekord, Iran \\ ${ }^{3}$ Department of Speech and Language Pathology, School of Rehabilitation Sciences, Iran University \\ of Medical Science, Tehran, Iran
}

\section{Abstract}

Introduction: The contents of the retropharyngeal space are limited to fat and retropharyngeal nodes. Primary tumors originating from the retropharyngeal space are rare, therefore, the existence of schwannoma in the retropharyngeal space is infrequent.

Method: This paper describes a case of a large schwannoma of the retropharyngeal space excised transorally with the use of co2 laser. This lesion measured about 6/6 x 3/5.

Result: No peri-or post-operative complications were encountered. The procedure allowed quick resumption of an oral diet and a return to normal activity for the patient.

Conclusion: This is, to our knowledge, the first report of this technique used in the excision of a large retropharyngeal space schwannoma.

\section{Introduction}

Schwannomais a benign slow-growing encapsulated nerve sheath tumor composed of Schwann cells in a collagenous matrix. That can arise from any cranial, peripheral, or autonomic nerve in the body. Approximately $25 \%-45 \%$ of all schwannomas occur in the head and neck, and most of these are found in the parapharyngeal space $[1,2]$. The retropharyngeal space only contains fat and the retropharyngeal lymph nodes; therefore, metastatic nodes from primary head and neck cancers account for the majority of tumor lesions in the retropharyngeal space. Primary tumors originating from the retropharyngeal space are rare; retropharyngeal lipoma, neuroblastoma, and pleomorphic adenoma have been reported only sporadically, therefore, the existence of schwannoma in the retropharyngealspace is infrequent [3]. Transcervical approach is used for complete enbloc removal of retropharyngeal tumor traditionally. However, neurogenic injury, unfavorable scars, loss of function, etc. are potential post-operative sequelae. The use of laser applications in otorhinolaryngology has undergone significant advances over

\author{
More Information \\ *Address for Correspondence: Zahra Danesh, \\ M.D, Otolaryngologist, Department of \\ Otolaryngology Head Neak Surgery, Kashani \\ Hospital, Parastar Street, Shahrekord, Iran, \\ Email: daneshzahra33@gmail.com \\ Submitted: March 19, 2021 \\ Approved: April 23, 2021 \\ Published: April 26, 2021
}

How to cite this article: Dabirmoghaddam $P$, Danesh Z, Danesh A. Transoral laser resection of a large schwannoma in the retropharyngeal space. J Clin Intensive Care Med. 2021; 6: 006-008.

DOI: 10.29328/journal.jcicm.1001033

ORCiD: orcid.org/0000-0001-7710-7576

Copyright: @ 2021 Dabirmoghaddam P, et al. This is an open access article distributed under the Creative Commons Attribution License, which permits unrestricted use, distribution, and reproduction in any medium, provided the original work is properly cited.

Keywords: Retropharyngeal Space; Schwannoma; Laser

\section{Check for updates}

OPEN ACCESS the past several years; laser technology is now used in a wide variety of procedures, and has become the primary treatment modality or standard of care for many otorhinolaryngology conditions. This procedure can be performed with fairly little bleeding, swelling, pain or scarring [4]. Here, we report a case whereby transoralco2laser excision was performed in a patient presented with large retropharyngeal schwannoma.

\section{Case presentation}

A 27-year-old man with no other significant medical history presented to our clinic for evaluation of neck pain. $\mathrm{He}$ also reported a slowly progressive neck deviation to right for last 3years.He had no complaint of dysphagia, odynophagia dyspnea and dysphonia. On physical examination there was an oval mass in the posterior hypopharyngeal wall which was covered by smooth and intact mucosa (Figure 1). Computed tomography (CT) of the neck revealed a large well defined tumor in the left retropharyngeal space measuring approximately $31 \times 45 \mathrm{~mm}$. The tumor was homogeneously hypoattenuated to the skeletal muscle, with no enhancement. Magnetic resonance imaging (MRI) showed a well-defined 
encapsulated tumor in the left retropharyngeal space measuring approximately $65 \times 35 \mathrm{~mm}$ with bright signal intensity on T2-weighted images and low signal intensity on T1-weighted images, which was heterogeneously enhanced after gadolinium administration (Figure 2). The patient opted for surgical excision via a endoscopic transoral laser or a possible transcervical route. Resection of the tumor was performed by $\mathrm{CO} 2$ laser through an endoscopic transoral approach under general anesthesia (Figure 3). During the surgery, a well encapsulated yellowish tumor measuring about $6 / 5 \times 3 / 5 \mathrm{~cm}$ was completely removed. The patient was kept non per so for 1 day and resumed an oral diet the following day. Postoperative course was uneventful and no sympathetic or cranial nerve dysfunction was has been occurred. Histopathological diagnosis was schwannoma (Figure 4).

\section{Discussion}

The retropharyngeal space extends from the clivus to the mediastinum and lies posterior to the pharynx and esophagus and anterior to the prevertebral muscles .It is bounded by the visceral fascia anteriorly, the prevertebral fascia posteriorly, and the carotid sheaths laterally. The retropharyngeal

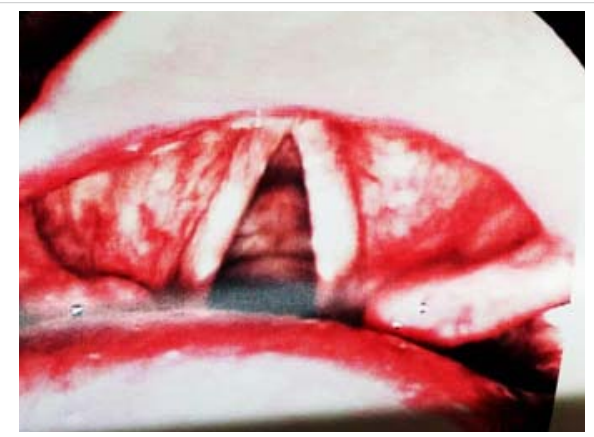

Figure 1: Videostrobuscopy shows an oval mass in the posterior hypopharyngeal wall which was covered by smooth and intact mucosa.

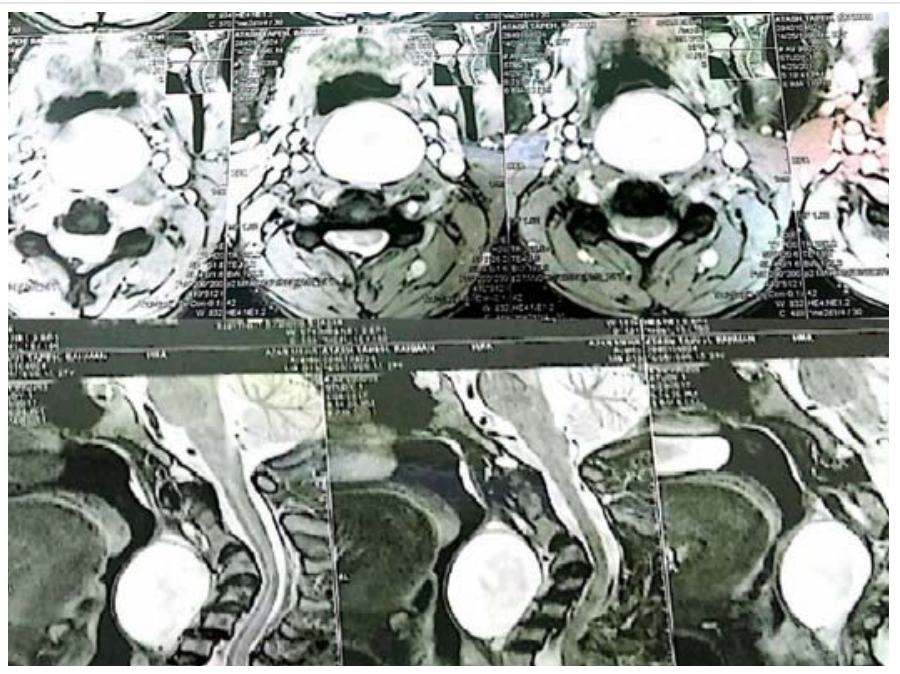

Figure 2: MRI showed a welldefined encapsulated tumor in the left retropharyngeal space measuring approximately $65 \times 35 \mathrm{~mm}$ with bright signal intensity on T2weighted images and low signal intensity on T1-weighted images, which was hetrogenously enhanced after gadolinium administration.

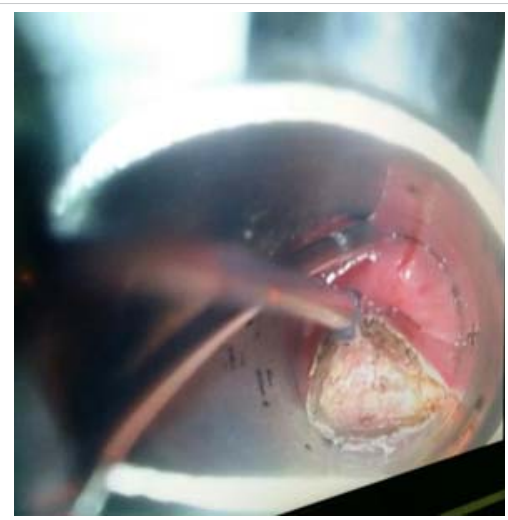

Figure 3: Intraoperative view of tumor resecton by $\mathrm{CO}_{2}$ laser.

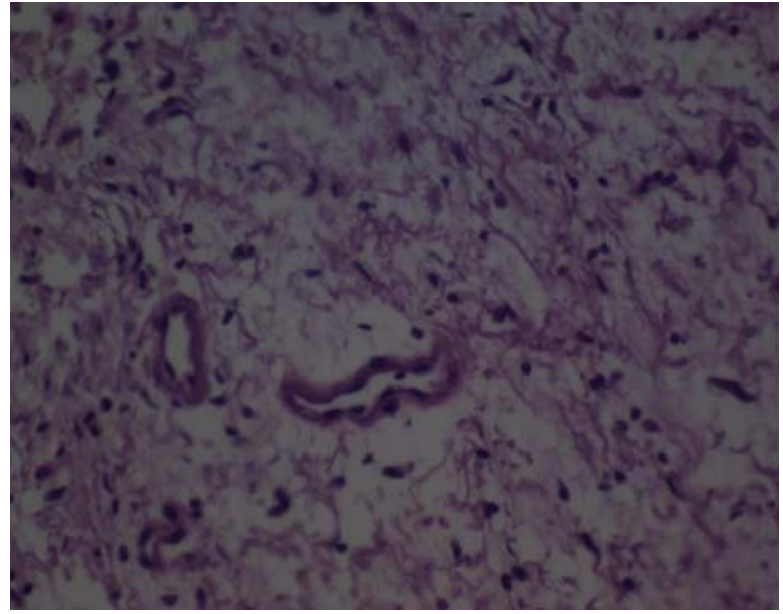

Figure 4: Final histologic examination which confirm diagnosis of schwannoma.

space can also be conceptually divided into the suprahyoid and infrahyoid retropharyngeal space. The suprahyoid retropharyngeal space contains fat and lymph nodes, while the infrahyoid retropharyngeal space contains predominantly fat [5]. The majority content of the retropharyngeal space is fat tissue; thus, lipoma appears to be the most common primary tumor in this space. Other tumors frequently seen are liposarcoma and lymphoid tumor (sarcoidosis, lymphomatous tumor, metatstatic lymph node). Schwannoma, on the other hand, has only been sporadically reported to occur in retropharyngeal space. This is because anatomically the major peripheral, cranial or autonomic nerves are more commonly found at parapharyngeal space, oral cavity and sinonasal regions, compared to retropharyngeal space [6]. Schwannomas originate from perineural Schwann cells and grow extrinsic to their parent nerve fascicles; they can occur along both sympathetic and somatic nerves in the body, with the exception of the olfactory and optic nerves, as these lack Schwann cells [7]. Schwannomas are mostly asymptomatic and the patient may occasionally present with constellation of symptoms such as dysphagia, airway compromise, globus sensation or rarely change in voice. If patient complains of associated paresthesia, neuralgia, rapid progression in size or worsening of symptoms, then malignant transformation should be suspected [8]. Among the imaging modalities, MRI with and without gadolinium enhancement is particularly 
helpful in delineating schwannoma. On MRI, schwannomas show low signal intensity on T1-weighted images and high signal intensity on T7-weighted images. Following the administration of gadolinium, the solid part of the tumor is strongly enhanced. The imaging helps to identify the site, size, extension and relation of the tumor with surrounding vessels and nervous system $[3,6]$.

The treatment is complete surgical excision of the tumor. Transcervical, transparotid and transoral excisions are the most reviewed surgical approaches in literature to remove retropharyngeal tumor [6].

CO2 lasers are widely used to remove thin layers from the surface of the mucosa without undermining the deeper layers. This procedure can be performed with fairly little bleeding, swelling, pain or scarring. The lasers are more precise than scalpels and the high temperature generated by the lasers aids in cleaning the edges of the body tissue that it is cutting, reducing the risk of infection and recurrence. Using this approach, the surgery time may be reduced and the healing time may be shortened. CO2 lasers now play an increasingly important role as a minimally invasive alternative to conventional surgical interventions for patients in a number of oncology services [4].

In our case MRI and CT showed that the tumor had a typical schwannoma appearance and may have originated from a small nerve supplying the pharynx, without involvement of the main trunk of the major nerves. The tumor resected by co 2 laser through an endoscopic transoral approach. This avoided the need for an external excision and the associated morbidity, which includes a long scar, longer hospital stay and associated pain. After operation, the surgical wound healed rapidly without causing swallowing problems, and the patient has remained free of any recurrence or neurologic deficits. This is, to our knowledge, the first report of this technique used in the excision of a large retropharyngeal space schwannoma.

\section{Result}

No peri-or post-operative complications were encountered. The procedure allowed quick resumption of an oral diet and a return to normal activity for the patient.

\section{Conclusion}

This is, to our knowledge, the first report of Transoral co2 Laser used in the excision of a large retropharyngeal space schwannoma.

\section{References}

1. Anil G, Tan TY. Imaging charecteristics of schwannOma of the sympathetic chain: A Review of 12 cases. AM J Neuroradiol. 2010: 31: 1408-1412.

PubMed: https://www.ncbi.nlm.nih.gov/pmc/articles/pmc7966108/

2. Bao YY, Yang H, He LY, Xu B. et al. Schwannoma of the epiglottis mimiking epiglottic cyst: A case presentation and literature review. Int $\mathrm{J}$ Clin Exp Med. 2017; 10: 3923-3927

3. Hsieh CY, Hsiao JK, Wang CP. Retropharyngeal schwannoma excised through a transoral approach: a case report. Kaohsiung J Med Sci. 2006; 22: 465-468.

4. Kou W, Zhang C, Wei P. Laryngeal schwannoma treated with a co2 laser: A case report. Oncology Letters 2015; 9: 1467-1469. PubMed: https://www.ncbi.nlm.nih.gov/pmc/articles/PMC4315079/

5. Hoang JK, Eastwood JD, Branst BF, et al. Masses in the retropharyngeal space: Kay concept on Multiplan CT and MR Imaging. Neurographics. 2011; 01: 49-55

6. Ngu CYV, Gan CC, Tang IP. Transoral excicion of retropharyngeal schwannoma: case report. ACTA Otolaryngologica Case Reports. 2017: 64-67.

7. Wang B, Yuan J, Chen X, Xu H, Zhou Y,, et al. Extracranial non vestibular head and neck schwannoma. Saudi Med J. 2015: 36: 1363-1366. PubMed: https://www.ncbi.nlm.nih.gov/pmc/articles/PMC4673378/

8. Ashish G, Mathew GA, Parmar H. Schwannoma of posterior pharyngeal wall: An unusual tumor in an unusual location. Otorhinolaryngol Clin: An Int J. 2016; 8: 35-37. 